\title{
Influencia de estilos de vida en el estado nutricional de estudiantes
}

universitarios

DOI: 10.17533/udea.penh.v20n2a03

PERSPECTIVAS EN NUTRICIÓN HUMANA

ISSN 0124-4108

Escuela de Nutrición y Dietética, Universidad de Antioquia. Medellín, Colombia

Vol. 20, N. ${ }^{\circ} 2$, julio-diciembre de 2018, p. 145-156.
Artículo recibido: 21 de junio de 2018

Aprobado: 22 de noviembre de 2018

\section{Ruth Adriana Yaguachi Alarcón”; Mariela Felisa Reyes López²; Carlos Luis Poveda Loor ${ }^{3}$}

\section{- -Resumen}

Antecedentes: el acceso a la universidad supone un cambio importante en el estilo de vida del estudiante, que puede repercutir en su estado nutricional. Objetivo: buscar una asociación entre estilo de vida y cambios antropométricos en estudiantes universitarios de la Escuela Superior Politécnica del Litoral de Guayaquil, Ecuador en un periodo de tres años. Materiales y métodos: estudio descriptivo de cohorte con una muestra de 336 estudiantes de ambos sexos en quienes se evaluó el estado nutricional por antropometría y se indagó sobre su estilo de vida mediante encuesta previamente validada. Resultados: entre 2014 y 2017 incrementó el exceso de peso (sobrepeso + obesidad) de 25,6 \% a 31,9\%, IMC, grasa corporal, circunferencia de cintura, circunferencia de cadera, e índice cintura/cadera $(p<0,05)$. Se asoció el consumo de alcohol con mayor peso $(+3,7 \mathrm{~kg})$ e índice cintura/cadera; $y$, mayor frecuencia de consumo de gaseosas con mayor peso $(+5 \mathrm{~kg})$. El consumo de comidas rápidas se asoció con el incremento del índice cintura/cadera $(p<0,05)$. No se encontraron diferencias antropométricas según consumo de cigarrillos, actividad física, consumo de frutas y verduras. Conclusión: en la población universitaria estudiada, el exceso de peso y de grasa corporal está aumentando; el consumo de alcohol, de gaseosas y fumar afectan el estado nutricional.

Palabras clave: estilo de vida, estado nutricional, estudiantes universitarios, sobrepeso, obesidad, conducta alimentaria.

$1^{*}$ Autor de correspondencia. Magíster en Nutrición Clínica. Nutricionista. Escuela Superior Politécnica del Litoral, ESPOL, Facultad Ciencias de la Vida, Campus Gustavo Galindo Km. 30.5 Vía Perimetral, P.O. Box 09-01-5863. Guayaquil-Ecuador. yaguachi@espol.edu.ec

2 Magíster en Administración de Negocios. Escuela Superior Politécnica del Litoral, ESPOL, Facultad Ciencias de la Vida, Campus Gustavo Galindo Km. 30.5 Vía Perimetral, P.O. Box 09-01-5863. Guayaquil-Ecuador. mfreyes@espol.edu.ec

3 Magíster en Procesamiento en Alimentos. Escuela Superior Politécnica del Litoral, ESPOL, Facultad Ciencias de la Vida, Campus Gustavo Galindo Km. 30.5 Vía Perimetral, P.O. Box 09-01-5863. Guayaquil-Ecuador. cpoveda@espol.edu.ec

Cómo citar este artículo: Yaguachi RA, Reyes MF, Poveda CL. Influencia de estilos de vida en el estado nutricional de estudiantes universitarios. Perspect Nutr Humana. 2018;20:145-56. DOI: 10.17533/udea.penh.v20n2a03 


\section{Influence of Lifestyles on the Nutritional Status of University Students}

\section{Abstract}

Background: Attendance to university is a major change in a student's lifestyle, which may be reflected in their nutritional status. Objective: Seek the association between lifestyle and anthropometric changes in university students of the Polytechnic School of the Litoral in Guayaquil-Ecuador over a period of three years. Materials and Methods: Descriptive cohort study with a sample of 336 students of both sexes, in whom nutritional status was evaluated by anthropometry, and lifestyle was investigated through a previously validated survey. Results: Between 2014 and 2017, excess weight (overweight + obesity) increased from $25.6 \%$ to $31.9 \%$, BMl, body fat, waist circumference, hip circumference and waist to hip ratio $(\mathrm{p}<0.05)$. Alcohol consumption was associated with greater weight $(+3.7 \mathrm{Kg})$ and waist to hip ratio, and higher frequency of consumption of soft drinks with greater weight $(+5 \mathrm{~kg})$. The consumption of fast foods was associated with increase in waist to hip ratio $(p<0.005)$. No anthropometric differences were found associated with cigarette use, physical activity, or consumption of fruits and vegetables. Conclusion: In the university population studied, excess weight and body fat is increasing, while alcohol and soda consumption as well as smoking affect nutritional status.

Keywords: Lifestyle, nutritional status, university students, overweight, obesity, feeding behavior.

\section{INTRODUCCIÓN}

La población universitaria constituye un grupo vulnerable de una serie de procesos fisiológicos característicos de la edad, a los que se suman posibles cambios socioculturales, ya que en muchas ocasiones deben dejar sus hogares (1). Estos factores influyen directamente en la modificación de su estilo de vida, debido a que se enfrentan a la oportunidad de tomar sus propias decisiones sobre los alimentos que van a consumir; esto podría tener repercusiones negativas a futuro sobre su salud e incrementar el riesgo de malnutrición (2).

Diversos autores han destacado que la población universitaria se caracteriza por la omisión de comidas y a su vez tienden a consumir alimentos poco saludables, pues en muchas ocasiones disponen de insuficiente tiempo para comer. Es por ello que durante este periodo se deberían desarrollar hábitos alimentarios saludables que ayuden a mejorar su estado nutricional y prevenir futuras enfermedades (3).

En Ecuador, la Encuesta Nacional de Salud y Nutrición (ENSANUT) demuestra que el consumo de kilocalorías promedio en hombres y mujeres adultos de 19 a 59 años es de 2143 y 1822 kcal respectivamente; se observa un mayor consumo en varones (4).

La contribución de las kilocalorías derivadas de los macronutrientes se encuentran dentro de los porcentajes normales. No obstante, el consumo de ácidos grasos saturados se encuentra por encima de los rangos recomendados (4).

Con respecto al tipo de alimentos consumidos, se muestra una alta tendencia al consumo de carbohidratos con un alto índice glicémico, como arroz blanco y pan. Entre el consumo de alimentos de alto aporte energético y bajo valor nutricional, se 
puede destacar que el consumo de gaseosas se encuentra entre los alimentos que más contribuyen al consumo diario de carbohidratos a nivel de Ecuador, así como de todas sus subregiones. Con relación al consumo de hamburguesas, tacos, salchipapas, perros calientes y papas fritas, se observa mayor consumo en el grupo de 15 a 19 años, $56,6 \%$ en hombres y 50,8 \% en mujeres (4). Para conocer cómo cambia el estado nutricional de la población universitaria y su relación con otras variables, se realizó un estudio cuyo objetivo fue buscar una asociación entre estilo de vida y cambios antropométricos en estudiantes universitarios de la Escuela Superior Politécnica del Litoral (ESPOL) de Guayaquil, Ecuador, en un periodo de tres años.

\section{MATERIALES Y MÉTODOS}

Se realizó un estudio descriptivo de cohorte. La muestra estuvo constituida por 336 estudiantes de la ESPOL de Guayaquil, Ecuador. Fueron elegibles para ser incluidos en la investigación los estudiantes que se realizaron la evaluación nutricional en el año 2014, previo al ingreso a la ESPOL, y que dieron su consentimiento para participar nuevamente en el año 2017. Se excluyeron mujeres en periodo de gestación o lactancia y personas con algún tipo de discapacidad física, que impidiera la toma de peso, talla y circunferencias.

\section{Evaluación antropométrica}

El peso en $\mathrm{kg}$ y el porcentaje de grasa corporal se determinaron mediante una balanza electrónica Tanita $^{\circledR}$ (SC-331S) debidamente calibrada; la persona estaba descalza y con la menor cantidad de ropa posible (5). La talla en $\mathrm{cm}$ se midió con un tallímetro marca $\mathrm{SECA}^{\circledR} 217$ con $1 \mathrm{~mm}$ de precisión; el estudiante estaba descalzo con los talones juntos; cabeza, hombros y glúteos erguidos. La toma de las circunferencias de cintura y cadera se realizó con una cinta métrica flexible de $1 \mathrm{~mm}$ de precisión. La medición de la circunferencia de cintura de cada participante se realizó con el abdomen descubierto, en posición erecta y relajada, realizándose al final de una espiración normal sin comprimir la cinta con la piel. Finalmente, la circunferencia de cadera se estableció con el sujeto de pie, con los brazos relajados y los pies juntos, se tomó a nivel de la máxima extensión de los glúteos (6).

La clasificación del estado nutricional según el Îndice de Masa Corporal (IMC) se hizo de acuerdo con los puntos de corte que se muestran en la tabla 1 (7). El índice cintura/cadera (ICC) permitió establecer la distribución de grasa corporal y en consecuencia el riesgo cardiovascular, que fue clasificado así: se determinó que un índice $\geq 0,8$ era indicativo de riesgo en la mujer; y en los hombres, un valor $\geq 1$ (8).

Tabla 1. Clasificación nutricional por el índice de masa corporal

\begin{tabular}{lc}
\hline Categoría & IMC $\mathbf{( \mathbf { k g } / \mathbf { m } ^ { 2 } )}$ \\
\hline Déficit energético grado III & $\leq 16$ \\
Déficit energético grado II & $16-16,9$ \\
Déficit energético grado I & $17-18,5$ \\
Normal & $18,5-24,9$ \\
Sobrepeso & $25,0-26,9$ \\
Preobesidad & $27,0-29,9$ \\
Obesidad de tipo I & $30,0-34,9$ \\
Obesidad de tipo II & $35,0-39,9$ \\
Obesidad de tipo III (mórbida) & $40,0-49,9$ \\
Obesidad de tipo IV (extrema) & $\geq 50$ \\
\hline
\end{tabular}

Fuente: Ladino et al. (7).

\section{Estilos de vida}

Para identificar los hábitos alimentarios se diseñó una encuesta de frecuencia de consumo de alimentos, tomando en cuenta para la construcción el cuestionario validado por García D, citado por Ladino (7), en el que se presentan los alimentos agrupados en 10 categorías y en el que los estu- 
diantes podían escoger una de las siguientes frecuencias de consumo: diario, semanal, quincenal, mensual o nunca. Con respecto a la ingesta de alcohol, se clasificó como abstemios a las personas que no consumían alcohol y solo en fiestas a quienes lo bebían en eventos sociales. El consumo de tabaco se clasificó de acuerdo con los parámetros establecidos por la OMS en el 2003, citado por Londoño (9); así, se consideró como fumadores leves a quienes fumaban menos de cinco cigarrillos al día; moderados a aquellos que fumaban un promedio de 6 a 15 cigarrillos diarios, y severos, a quienes fumaban más de 16 cigarrillos por día.

La actividad física se determinó por recordatorio, considerando el tipo de actividad física realizada, duración en minutos/horas y su frecuencia diaria o semanal. Posteriormente, siguiendo la metodología propuesta por el Institute of Medicine of The National Academies, se calculó el nivel de actividad diaria de cada sujeto, con los puntos de corte que se muestran en la tabla 2 (8).

Tabla 2. Clasificación del grado de actividad física

\begin{tabular}{lc}
\hline Clasificación & Nivel de actividad física \\
\hline Ligera & $1,0<1,4$ \\
Moderada & $1,4<1,6$ \\
Vigorosa & $>1,6$ \\
\hline
\end{tabular}

Fuente: Mahan LK et al. (8).

\section{Análisis estadístico}

Los datos sociodemográficos, antropométricos y de estilo de vida fueron almacenados en un contenedor digital construido sobre Excel para Office de Windows ${ }^{\circledR}$. Se empleó el paquete estadístico SPSS ${ }^{\circledR}$ versión 23 para el procesamiento de los datos y el análisis de los resultados.

De acuerdo con la naturaleza de cada variable, se realizó un análisis descriptivo, se calcularon las medidas de tendencia central, como la media y las medidas de dispersión como la desviación estándar y el rango. Se realizó la prueba Wilcoxon a los datos obtenidos de los participantes durante los dos periodos de tiempo para determinar la significancia estadística de cada una de ellos. Para establecer la asociación entre el estilo de vida y el estado nutricional actual de los estudiantes universitarios, se aplicó las pruebas estadísticas $U$ de Mann-Whitney y Kruskall-Wallis.

\section{Consideraciones éticas}

El proyecto fue aprobado por el Decanato de Investigación de la ESPOL. Todos los participantes recibieron información acerca del estudio y firmaron de manera voluntaria un consentimiento informado. Se siguieron las normas bioéticas establecidas por la Declaración de Helsinki (10).

\section{RESULTADOS}

El grupo de estudio estuvo conformado por 147 varones y 189 mujeres que representaron el $43,7 \%$ y $56,3 \%$ respectivamente. La edad estuvo comprendida entre 19 y 33 años, con un promedio de 20,5 $\pm 1,0$ años.

Al comparar el estado nutricional de los evaluados durante los dos periodos, 2014 y 2017 (Tabla 3), se observó que el mayor porcentaje de investigados presentó un IMC dentro de los valores normales $\left(18,5\right.$ a $\left.24,9 \mathrm{~kg} / \mathrm{m}^{2}\right)$. Al agrupar los valores de sobrepeso y obesidad, se obtuvo una prevalencia de exceso de peso de $25,6 \%$ en el 2014; para el 2017 se incrementó a 31,9\%. Con respecto a la desnutrición en sus diferentes grados, se muestra que disminuyó de 8,1 a 6,0 \%.

En relación con el porcentaje de grasa corporal, la mayoría de evaluados presentó un nivel aceptable tanto en el 2014 (27,7 \%) como en el 2017 (33,3\%). Un menor porcentaje mostró obesidad de acuerdo con el porcentaje de grasa corporal. 
Tabla 3. Evaluación antropométrica del estado nutricional y de riesgo cardiometabólico según año de evaluación

\begin{tabular}{|c|c|c|c|c|c|}
\hline & & \multicolumn{4}{|c|}{ Año } \\
\hline \multicolumn{2}{|c|}{ Indicadores antropométricos } & \multicolumn{2}{|c|}{2014} & \multicolumn{2}{|c|}{2017} \\
\hline & & $\mathrm{N}^{\mathrm{a}}$ & $\%$ & $\mathrm{~N}^{\mathrm{a}}$ & $\%$ \\
\hline \multirow{9}{*}{ IMC $\left(\mathrm{kg} / \mathrm{m}^{2}\right)$} & Déficit energético grado III & 5 & 1,5 & 15 & 4,5 \\
\hline & Déficit energético grado II & 2 & 0,6 & 3 & 0,9 \\
\hline & Déficit energético grado I & 20 & 6 & 2 & 0,6 \\
\hline & Normal & 223 & 66,4 & 209 & 62,2 \\
\hline & Sobrepeso & 46 & 13,7 & 55 & 16,4 \\
\hline & Preobesidad & 23 & 6,8 & 27 & 8 \\
\hline & Obesidad de tipo I & 15 & 4,5 & 19 & 5,7 \\
\hline & Obesidad de tipo II & 2 & 0,6 & 5 & 1,5 \\
\hline & Obesidad de tipo III (mórbida) & 0 & 0 & 1 & 0,3 \\
\hline \multirow{5}{*}{ Grasa corporal (\%) } & Aceptable & 93 & 27,7 & 112 & 33,3 \\
\hline & Buena & 84 & 25 & 74 & 22 \\
\hline & Muy buena & 60 & 17,9 & 43 & 1,8 \\
\hline & Sobrepeso & 66 & 19,6 & 76 & 22,6 \\
\hline & Obesidad & 33 & 9,8 & 31 & 9,2 \\
\hline \multirow{2}{*}{ ICC } & Con riesgo cardiometabólico & 44 & 13,1 & 43 & 12,8 \\
\hline & Sin riesgo & 292 & 86,9 & 293 & 87,2 \\
\hline
\end{tabular}

De acuerdo con el ICC, se reflejó que el mayor porcentaje de investigados no presentaba riesgo cardiovascular (2014: 86,9 \% y 2017: 87,2\%).

En la tabla 4, se muestran las características antropométricas de los estudiantes evaluados durante los dos periodos de tiempo. Se observó que el peso se incrementó en promedio $2 \mathrm{~kg}$ al pasar de $61,8 \pm 12,8 \mathrm{~kg}$ en 2014 a $63,8 \pm 13,7 \mathrm{~kg}$ en 2017 $(p<0,05)$. En cuanto al IMC promedio se encontró un ligero incremento inferior a $1 \mathrm{~kg}$ : pasó de 23,0 $\pm 3,7$ a $23,7 \pm 4,0 \mathrm{~kg}(\mathrm{p}<0,05)$, al igual que el porcentaje de grasa corporal promedio que pasó de $20,0 \pm 8,4$ a $21,2 \pm 8,2$ entre el 2014 y el $2017(\Delta=+1,2 ; p<0,05)$. Por otro lado, se elevó tanto la circunferencia cintura de $78,3 \pm 9,8$ a $79,9 \pm 10,6(\Delta=+1.6 ; p<0,05)$ como la de cadera, de 99,5 \pm 8,3 a 100,6 $48,3(\Delta=+1,1$; $p<0,05)$. Asimismo, se evidenció un ligero incremento, pero significativo de ICC (2014: 0,78 $\pm 0,05$ vs 2017: $0,79 \pm 0,06 ; \Delta=+0,01 ; p<0,05)$. En la tabla 5 , se muestra la variable de estilo de vida según estado nutricional medido por antropome- tría. Se evidenció que la ingesta social de alcohol en comparación con los abstemios se asoció tanto con un mayor peso (abstemio: $62,2 \pm 13,4$ vs. solo en fiestas: $65,9 \pm 13,8 ; \Delta=+3,7 ; p<0,05)$ como con un ICC de $0,78 \pm 0,06$ a $0,80 \pm 0,07$ más alto $(\Delta=+0,02 ; p<0,05)$. Finalmente, no se encontraron diferencias entre los parámetros antropométricos con el consumo de cigarrillos y los distintos grados de actividad física.

Al relacionar el consumo de alimentos con la evaluación antropométrica (Tabla 6), se pudo determinar que el consumo de comidas rápidas con una frecuencia diaria y semanal se relacionó con el incremento del ICC $(p<0,05)$. Del mismo modo, el consumo de bebidas gaseosas con una frecuencia diaria y semanal se asoció con un peso más elevado $(p<0,05)$, una circunferencia de cintura más alta y mayor circunferencia de cadera, lo mismo que el ICC. Para la frecuencia de consumo de verduras, frutas y productos de bollería (panadería), no se evidenció asociación. 
Estilos de vida en estudiantes universitarios

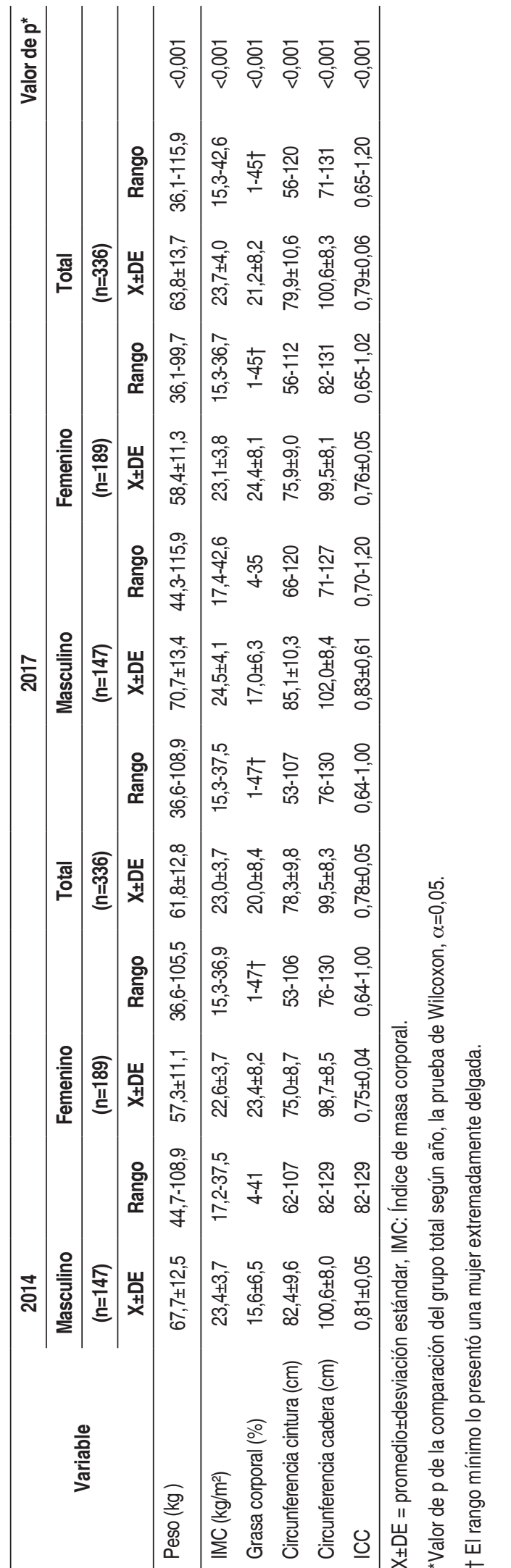

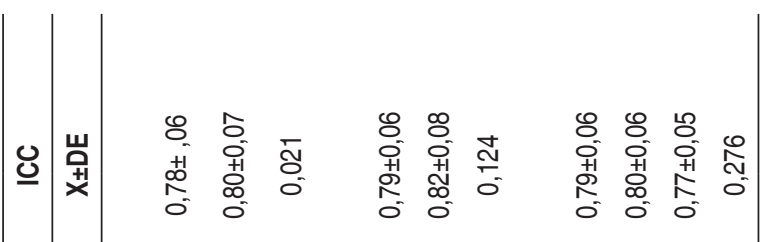

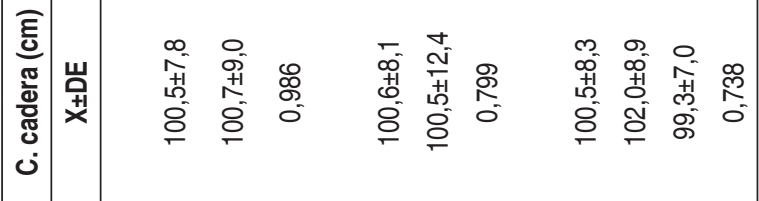

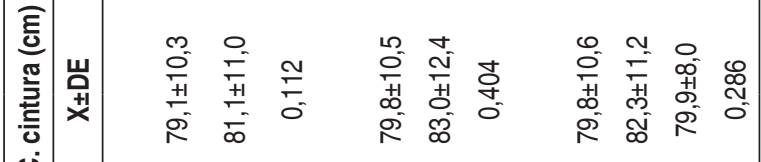

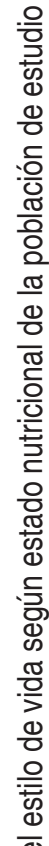

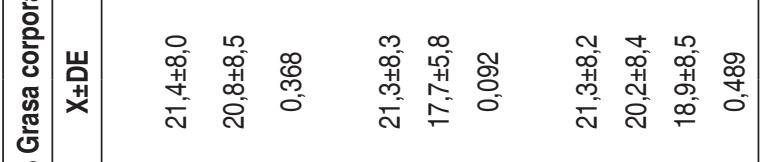

ஃ̊

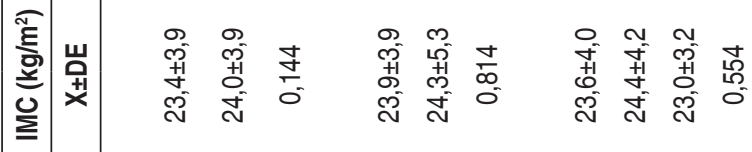

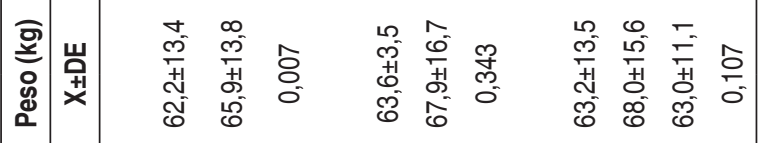

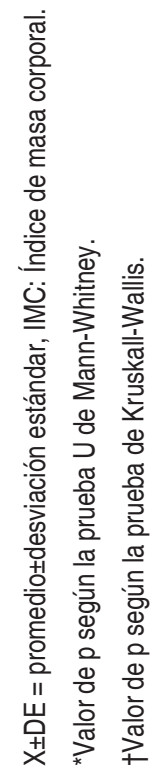


Tabla 6. Distribución del consumo de alimentos según estado nutricional

\begin{tabular}{|c|c|c|c|c|c|c|c|}
\hline \multicolumn{2}{|c|}{ Consumo de alimentos } & \multirow{2}{*}{$\begin{array}{c}\text { Peso (kg) } \\
X \pm D E\end{array}$} & \multirow{2}{*}{$\begin{array}{c}\operatorname{IMC}\left(\mathrm{k} / \mathrm{m}^{2}\right) \\
\mathrm{X} \pm \mathrm{DE} \\
\end{array}$} & \multirow{2}{*}{$\begin{array}{c}\text { \% Grasa corporal } \\
\mathrm{X} \pm \mathrm{DE} \\
\end{array}$} & \multirow{2}{*}{$\begin{array}{c}\text { C. cintura (cm) } \\
X \pm D E \\
\end{array}$} & \multirow{2}{*}{$\begin{array}{c}\text { C. cadera (cm) } \\
X \pm D E \\
\end{array}$} & \multirow{2}{*}{$\begin{array}{c}\text { ICC } \\
\mathrm{X} \pm \mathrm{DE} \\
\end{array}$} \\
\hline \multirow{5}{*}{ Verduras } & & & & & & & \\
\hline & Diario (n=173) & $63,9 \pm 13,4$ & $23,8 \pm 4,2$ & $21,1 \pm 8,7$ & $80,0 \pm 10,7$ & $100,6 \pm 8,4$ & $0,79 \pm 0,06$ \\
\hline & Semanal $(n=144)$ & $63,6 \pm 14,1$ & $23,5 \pm 3,8$ & $21,4 \pm 7,6$ & $79,5 \pm 10,6$ & $100,7 \pm 8,3$ & $0,79 \pm 0,06$ \\
\hline & Rara vez $(n=19)$ & $63,9 \pm 139$ & $23,3 \pm 4,3$ & $19,8 \pm 8,0$ & $80,5 \pm 11,1$ & $99,9 \pm 8,2$ & $0,80 \pm 0,07$ \\
\hline & Valor de $\mathrm{p}^{*}$ & 0.892 & 0,680 & 0,798 & 0,843 & 0,759 & 0,623 \\
\hline \multirow{4}{*}{ Frutas } & Diario $(n=144)$ & $64,5 \pm 13,5$ & $24,0 \pm 4,1$ & $21,8 \pm 8,3$ & $80,0 \pm 10,4$ & $100,9 \pm 7,9$ & $0,79 \pm 0,06$ \\
\hline & Semanal $(n=169)$ & $63,9 \pm 14,2$ & $23,6 \pm 4,0$ & $20,8 \pm 8,2$ & $80,1 \pm 8,5$ & $100,8 \pm 8,7$ & $0,79 \pm 0,06$ \\
\hline & Rara vez $(n=23)$ & $58,9 \pm 9.6$ & $22,3 \pm 2,9$ & $20,2 \pm 7,7$ & $76,6 \pm 8,5$ & $97,5 \pm 7,6$ & $0,78 \pm 0,08$ \\
\hline & Valor de $\mathrm{p}^{*}$ & 0,221 & 0,236 & 0,552 & 0,407 & 0,422 & 0,604 \\
\hline \multirow{4}{*}{ Comidas rápidas } & Diario $(n=44)$ & $63,2 \pm 14,0$ & $23,4 \pm 4,3$ & $20,0 \pm 9,1$ & $80,5 \pm 11,0$ & $101,0 \pm 9,2$ & $0,79 \pm 0,06$ \\
\hline & Semanal $(n=177)$ & $65,2 \pm 14,0$ & $24,0 \pm 4,0$ & $21,1 \pm 8,0$ & $81,0 \pm 10,8$ & $101,0 \pm 8,4$ & $0,79 \pm 0,06$ \\
\hline & Rara vez $(n=115)$ & $61,9 \pm 12,9$ & $23,2 \pm 4,0$ & $21,7 \pm 8,1$ & $78,1 \pm 10,0$ & $99,5 \pm 7,7$ & $0,78 \pm 0,07$ \\
\hline & Valor de $p^{*}$ & 0,143 & 0,207 & 0,266 & 0.073 & 0,503 & 0,048 \\
\hline \multirow{4}{*}{ Productos de bollería } & Diario $(n=42)$ & $64,5 \pm 14,2$ & $23,6 \pm 4,4$ & $20,3 \pm 8,0$ & $79,7 \pm 11,4$ & $100,9 \pm 8,7$ & $0,78 \pm 0,05$ \\
\hline & Semanal $(n=165)$ & $64,7 \pm 14,3$ & $23,9 \pm 4,1$ & $21,1 \pm 8,0$ & $81,0 \pm, 11,0$ & $101,0 \pm 8,2$ & $0,80 \pm 0,07$ \\
\hline & Rara vez $(n=129)$ & $62,4 \pm 12,6$ & $23,4 \pm 3,8$ & $21,5 \pm 8,6$ & $78,6 \pm 9,8$ & $100,1 \pm 8,3$ & $0,78 \pm 0,06$ \\
\hline & Valor de $\mathrm{p}^{*}$ & 0,557 & 0,751 & 0,628 & 0,212 & 0,381 & 0,135 \\
\hline \multirow{4}{*}{ Bebidas gaseosas } & Diario $(n=42)$ & $65,4 \pm 13,8$ & $23,9 \pm 4,4$ & $19,7 \pm 8,9$ & $81,9 \pm 12,0$ & $101,0 \pm 8,9$ & $0,80 \pm 0,08$ \\
\hline & Semanal $(n=159)$ & $66,3 \pm 14,5$ & $24,2 \pm 4,2$ & $21,0 \pm 8,5$ & $82,0 \pm 11,0$ & $102,0 \pm 8,5$ & $0,80 \pm 0,06$ \\
\hline & Rara vez $(n=135)$ & $60,2 \pm 11,9$ & $23,0 \pm 3,5$ & $21,8 \pm 7,6$ & $76,9 \pm 8,9$ & $98,9 \pm 7,5$ & $0,77 \pm 0,06$ \\
\hline & Valor de $\mathrm{p}^{*}$ & $<0,001$ & 0,061 & 0.108 & $<0,001$ & 0,015 & $<0,001$ \\
\hline
\end{tabular}

*Valor de $p$ según la prueba de Kruskall-Wallis

\section{DISCUSIÓN}

El presente trabajo evidencia la variación de los indicadores antropométricos de la población universitaria durante un periodo de 3 años (2014-2017) asociados con el estilo de vida.

Los resultados reflejan un incremento de la prevalencia de sobrepeso y obesidad del 6,3\% (2014: $25,6 \%$ y $2017: 31,9 \%$ ). Estos valores no se ven tan alejados de los datos reportados a nivel de Ecuador para este grupo de edad, siendo la prevalencia de sobrepeso y obesidad en mayores de 19 a 29 años de 45,0 \% y 47,9 \% en hombres y mujeres respectivamente (4). Resultados similares fueron reportados por la OMS en el año 2016, en los que el $39 \%$ de los adultos de 18 o más años padecían sobrepeso. Al mismo tiempo, la prevalencia de obesidad fue del $13 \%$, casi triplicándose a nivel mundial entre 1975 y 2016 (11). 
El IMC es el más utilizado para identificar a los adultos con sobrepeso y obesidad por su alta correlación de morbi-mortalidad en individuos de diversa distribución geográfica, estructura social y grupo de edad (12). Sin embargo, se han planteado varias limitaciones del IMC como indicador de obesidad, ya que no distingue la masa magra de la grasa en el peso corporal (13). Por lo tanto, para hablar de obesidad no solo se debe evaluar el estado nutricional del adulto a través del IMC, sino también determinar el porcentaje de grasa; es por ello que en el presente estudio se utilizó el porcentaje de grasa para medir los cambios de la composición corporal de los estudiantes universitarios en el transcurso de tiempo. Si bien es cierto que el mayor porcentaje de investigados presentó un nivel aceptable de grasa corporal en los años de evaluación (2014 y 2017), también se observa una ligera tendencia al incremento, especialmente del sobrepeso en un $3 \%$; aunque las causas se podrían relacionar con un exceso en el consumo de energía (ingesta dietética) en relación con su gasto. Su etiología en muchas ocasiones incluye factores genéticos, fisiológicos, ambientales, psicológicos, sociales, económicos e incluso políticos (14).

El incremento del ICC ha demostrado una asociación con un mayor riesgo de enfermedad cardiovascular (13). Se utiliza para detectar posibles signos de acumulación de grasa. Este índice ha sido ampliamente utilizado en estudios epidemiológicos realizados en Europa y Estados Unidos (15). Con relación al ICC, se estableció que un menor número de investigados presentaron riesgo cardiovascular asociado con hipercortisolismo, hipercolesterolemia, hipertensión, resistencia a la insulina y condiciones conductuales y sociales (6).

Al determinar las posibles causas en la variación del peso y demás indicadores antropométricos en la población universitaria en estudio, se determinó que la ingesta de alcohol solo en fiestas se asoció con mayor peso, en un promedio de
$3,7 \mathrm{~kg}(\mathrm{p}<0,05)$ y con un ICC ligeramente más alto $(0,02)$. El alcohol constituye una de las sustancias psicoactivas que, al igual que el cigarrillo, son usadas en mayor medida por adolescentes y jóvenes (16). En este sentido, los hombres la consumen con mayor frecuencia y tienen una marcada tendencia por las bebidas con una alta concentración de alcohol, como el aguardiente y el ron, aunque la más ingerida por los consumidores es la cerveza (17).

Paredes et al. (18) refirieron que "el alcohol aporta calorías vacías ( $1 \mathrm{~g}$ de etanol= $7 \mathrm{kcal}$ ), es decir, desprovistas de nutrientes como vitaminas y minerales" (p. 7), lo que constituye uno de los principales factores de riesgo para el desarrollo de enfermedades y lesiones como trastornos cardiometabólicos, enfermedades del hígado, dislipidemias, trastornos afectivos, entre otros (4). Varios autores han destacado la relación entre la ingesta de alcohol y el aumento de peso. En una investigación realizada en el municipio de Floridabanca, Colombia, con el objetivo de determinar la prevalencia de sobrepeso y obesidad en estudiantes adolescentes, se encontró asociación entre el consumo de alcohol y el sobrepeso (OR 4,1, IC $95 \% 1,1-15,5 ; p=0,009)$ (19). Estos resultados se contraponen a los encontrados en el estudio "Efectos de un consumo moderado de cerveza sobre la composición corporal", en el cual se sometió a un grupo de individuos a un consumo moderado de cerveza diario por 30 días y se evaluaron los parámetros antropométricos como peso, talla, pliegues cutáneos y perímetros. Como resultado no se evidenció cambios en los parámetros de composición corporal, a excepción del pliegue bicipital en hombres (20). Por las repercusiones que el consumo de alcohol puede tener en la salud de la persona, la OMS recomienda poner en práctica programas de tamizaje e intervenciones breves para disminuir su consumo peligroso y nocivo (21). 
La ingestión de bebidas alcohólicas está fuertemente asociada con el consumo de tabaco (18). De acuerdo con la OMS (22) "el consumo de tabaco mata cada año a más de 7 millones de personas, de las que más de 6 millones son consumidores del producto y alrededor de 890000 son no fumadores expuestos al humo de tabaco ajeno". Además, incrementa el riesgo de cáncer, enfermedades respiratorias y cardiovasculares (23). En la presente investigación no se encontraron diferencias antropométricas con el consumo de cigarrillo. La relación entre fumar y la obesidad es muy compleja (23), algunos estudios no han evidenciado asociación entre el tabaquismo y el IMC (24). Sin embargo, otros han indicado que fumar puede estar relacionado con un IMC bajo (25); mientras que dejar de fumar, con un IMC elevado (26). En un estudio transversal que contó con la participación de 499504 adultos de mediana edad en la población general de Reino Unido, se exploró la relación entre el tabaquismo y la obesidad; se determinó que los fumadores actuales tenían menos probabilidades de ser obesos que los que nunca habían fumado, mientras que los exfumadores tenían más probabilidades de ser obesos (23).

En el presente estudio, la actividad física no influyó en los resultados de los diferentes indicadores antropométricos. Sin embargo, se debe resaltar que existen diferencias entre los datos reportados con respecto al tipo de actividad física. En este sentido, los estudiantes que realizaban actividad física vigorosa tenían valores antropométricos inferiores en relación con aquellos que la practicaban con una intensidad ligera y moderada. A menudo, los estudiantes universitarios pueden presentar una disminución en los niveles de la actividad física, por cambios producidos en los estilos de vida durante la transición de la etapa escolar (27).

En un reciente estudio de tipo transversal, se determinó que el $51,38 \%$ de los estudiantes universitarios investigados se situaba por debajo de las recomendaciones internacionales de actividad física saludable (28). Según la OMS, la inactividad física constituye el cuarto factor de riesgo en lo que respecta a la mortalidad mundial (29), y al menos un $60 \%$ de la población mundial no realiza la actividad física necesaria para obtener beneficios para la salud (30). En Ecuador, según los datos reportados por Freire et al. (4), "alrededor de un cuarto $(24,6 \%$ ) de adultos de 18 hasta los 60 años son inactivos y más de un tercio tiene baja actividad física (34,6\%), mientras el 40,8\% reporta un nivel de mediana 0 alta actividad" (p. 593). Actualmente, se requiere que los países implementen medidas sanitarias que ayuden a incrementar los niveles de actividad física diaria en todos los grupos etarios de la población (30); es por ello que la OMS (31) sugiere que "los adultos aumenten hasta 300 minutos semanales su actividad física mediante ejercicios aeróbicos de intensidad moderada o practicar 150 minutos de actividad aeróbica vigorosa, o bien una combinación equivalente de actividad física moderada y vigorosa" (p. 8).

Otros de los factores estudiados para determinar las causas en la variación de los indicadores antropométricos en la población universitaria investigada fueron los hábitos alimentarios. Se estudió principalmente la frecuencia de consumo de verduras, frutas, productos de bollería (panadería), comidas rápidas y bebidas gaseosas; se encontró una relación significativa con estas dos últimas. El consumo diario y semanal de comidas rápidas se relacionó con el incremento del ICC $(p<0,05)$. Por otra parte, la ingestión de bebidas gaseosas con una frecuencia diaria y semanal se asoció con la ganancia de peso $(p<0,05)$, mayor circunferencia de cintura y de cadera e ICC.

De acuerdo con los datos reportados por Freire et al. (4) "las gaseosas, por su alto contenido de azúcar, se encuentran entre los alimentos que más contribuyen al consumo diario de carbohidratos a nivel de Ecuador, así como de todas sus 
subregiones" (p. 317). El consumo excesivo de bebidas gaseosas y comidas rápidas por su alto contenido calórico se asocia a un mayor IMC y, en consecuencia, con el incremento de peso, con factores asociados con diabetes, hipertensión y síndrome metabólico, entre otros. En el caso de los estudiantes universitarios, estos prefieren consumir alimentos poco saludables como gaseosas, perros calientes y empanadas por su bajo costo económico (32). En el estudio "Calidad de la alimentación y estado nutricional en estudiantes de 11 regiones de Chile", Ratner et al. (33) "observaron una alta frecuencia de consumo de pasteles, galletas, dulces, bebidas gaseosas y frituras. Además de una baja frecuencia de consumo diario de frutas, verduras y lácteos" (p. 1574).

Si bien es cierto que no se encontró una asociación significativa entre el consumo de frutas y verduras con la variación de los indicadores antropométricos en los investigados, es importante promover su consumo a diario en los estudiantes universitarios, por ser fuente de vitaminas, minerales, fibra y agua, y por tener baja densidad calórica (6). Además, constituyen componentes esenciales de una dieta saludable y podría contribuir a la prevención de enfermedades cardiovasculares y de algunos tipos de cáncer (34).

\section{CONCLUSIONES}

En la población universitaria estudiada, el exceso de peso y grasa corporal está aumentando; el consumo de alcohol, gaseosas y fumar afectan el estado nutricional.

Resulta indispensable la identificación temprana de los inadecuados estilos de vida que pudieran afectar el estado nutricional actual de los estudiantes universitarios para prevenir a futuro la ganancia de peso y enfermedades asociadas y, a su vez, permitiría crear estrategias de prevención y mejorar su calidad de vida a través de la educación alimentaria nutricional.

\section{CONFLICTO DE INTERESES}

Se declara que el manuscrito no contiene material protegido por derecho de reproducción ni genera conflicto de intereses.

\section{CONTRIBUCIONES DE LOS AUTORES}

Se declara que todos los autores tienen contribución equitativa en el desarrollo de la investigación y escritura del manuscrito.

\section{AGRADECIMIENTOS}

A la Escuela Superior Politécnica del Litoral (ESPOL) por el apoyo brindado durante la ejecución de la presente investigación.

El equipo de trabajo de la presente investigación extiende sus más sinceros agradecimientos a esta prestigiosa Revista y a sus expertos, por el apoyo incondicional durante todo el proceso de revisión del manuscrito. 


\section{-Referencias}

1. Ledo-Varela M, Román de L, González-Sagrado M, Izaola O, Conde V, Fuente A. Características nutricionales y estilo de vida en universitarios. Nutr Hosp. 2011;26(4):814-8.

2. Deshpande S, Basil MD, Basil DZ. Factors Influencing Healthy Eating Habits Among College Students: An Application of the Health Belief Model. Health Mark Q. 2009;26(2):145-64. DOI: 1080/07359680802619834

3. Ratner R, Hernández J, Martel J, Atalah E. Calidad de la alimentación y estado nutricional en estudiantes universitarios de 11 regiones de Chile. Rev méd Chile. 2012;140(12):1571-9. DOI: 10.4067/S0034-98872012001200008

4. Freire WB, Ramírez-Luzuriaga MJ, Belmont P, Mendieta MJ, Silva-Jaramillo MK, Romero N, et al. Tomo I: Encuesta Nacional de Salud y Nutrición de la población ecuatoriana de cero a 59 años. ENSANUT-ECU 2012. Ministerio de Salud Pública/ Instituto Nacional de Estadisticas y Censos. Quito-Ecuador; 2014.

5. Bezares V, Cruz R, Burgos M, Barrera M. Evaluación del estado de nutrición en el ciclo vital humano. 1. ${ }^{a}$ ed. México: McGraw-Hill; 2014.

6. Suverza A, Haua K. El ABCD de la evaluación del estado de nutrición. 1. a ed. México: Graw-Hill; 2010.

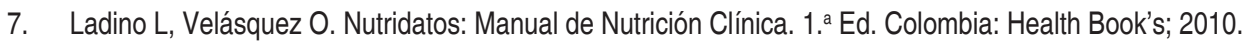

8. Mahan LK, Escott-Stump S, Raymond JL. Krause Dietoterapia. 13. ${ }^{a}$ ed. España: Elsevier; 2012.

9. Londoño C, Rodríguez I, Gantiva C. Cuestionario para la clasificación de consumidores de cigarrillo (C4) para jóvenes. Divers Perspect Psicol. 2011;7(2):281-91.

10. Asociación Médica Mundial (WMA). Declaración de Helsinki de la AMM - Principios éticos para las investigaciones médicas en seres humanos. 2017. [Internet]. [Citado marzo de 2018]. Disponible en: https://www.wma.net/es/policies-post/declaracion-de-helsinki-de-la-amm-principios-eticos-para-las-investigaciones-medicas-en-seres-humanos/

11. OMS. Obesidad y sobrepeso. 2017. [Internet]. [Citado marzo de 2018]. Disponible en: http://www.who.int/mediacentre/factsheets/fs311/es/

12. Gómez A. Evaluación del estado nutricional del adulto mediante la antropometría. Revista Cubana Aliment Nutr. 2012;16(2):146-52.

13. Moreno M. Definición y clasificación de la obesidad. Rev Médica Clínica Las Condes. 2012;23(2):124-8.

14. Wright S, Aronne LJ. Causes of obesity. Abdominal Radiology. 2012;37(5):730-2. DOI: 10.1007/s00261-012-9862-x

15. De Oliveira M, Fagundes R, Moreira E, De Moraes E, De Carvalho T. Relación de Indicadores Antropométricos con Factores de Riesgo para Enfermedad Cardiovascular. Arq Bras Cardiol. 2010;94(4):462-9.

16. Pérez $C$, Vinaccia S. Prevención del abuso en el consumo de alcohol en jóvenes universitarios: lineamientos en el diseño de programas costo-efectivos. Psicología y Salud. 2014;15(2):241-9.

17. Londoño $\mathrm{C}$, Valencia $\mathrm{C}$. Asertividad, resistencia a la presión de grupo y consumo de alcohol en universitarios. Acta Colombiana de Psicología. 2008;11(1):155-62.

18. Paredes R, Orraca O, Marimón E, Casanova M, Veliz D. Influencia del tabaquismo y el alcoholismo en el estado de salud de la población pinareña. Rev Ciencias Médicas Pinar Río. 2015;19(1):46-55.

19. Gamboa E, López N, Quintero D. Prevalencia de sobrepeso y obesidad en adolescentes del municipio de Floridablanca, Colombia. MedUNAB. 2007;10(1):5-12. DOI: 10.29375/01237047.120 
Estilos de vida en estudiantes universitarios

20. Romeo J, González-Gross M, Wärnberg J, Díaz L, Marcos A. ¿Influye la cerveza en el aumento de peso?: Efectos de un consumo moderado de cerveza sobre la composición corporal. Nutr Hosp. 2007;22(2):223-8.

21. OMS. Alcohol. 2015. [Internet]. [Citado abril de 2018]. Disponible en: http://www.who.int/mediacentre/factsheets/fs349/es/

22. OMS. Tabaco. [Internet]. [Citado abril de 2018]. Disponible en: http://www.who.int/mediacentre/factsheets/fs339/es/

23. Dare S, Mackay D, Pell J. Relationship between Smoking and Obesity: A Cross-Sectional Study of 499,504 Middle-Aged Adults in the UK General Population. PloS one. 2015;10(4). DOI: 10.1371/journal.pone.0123579

24. Zbikowski S, Jack LM, McClure JB, Deprey M, Javitz HS, McAfee TA, et al. Utilization of Services in a Randomized Trial Testing Phone- and Web-Based Interventions for Smoking Cessation. Nicotine \&Tobacco Research. 2011;13(5):319-27. DOI: $10.1093 / n t r / n t q 257$

25. Gritz E, Klesges R, Meyers A. The Smoking and Body Weight Relationship: Implications for Intervention and Postcessation Weight Control. Ann Behav Med. 1989;11(4):144-53. DOI: 1207/s15324796abm1104_4

26. Munafò M, Tilling K, Ben-Shlomo Y. Smoking status and body mass index: A longitudinal study. Nicotine \& Tobacco Research. 2009;11(6):765-71. DOI:10.1093/ntr/ntp062

27. Varela-Mato V, Cancela J, Ayan C, Martín V, Molina A. Lifestyle and Health among Spanish University Students: Differences by Gender and Academic Discipline. Int J Environ Res Public Health. 2012;9(8):2728-41. DOI: 10.3390/ijerph9082728

28. Práxedes A, Sevil J, Moreno A, Del Villar F, García-González L. Niveles de actividad física en estudiantes universitarios: diferencias en función del género, la edad y los estados de cambio. Revista iberoamericana de psicología del ejercicio y el deporte. 2016;11(1).

29. OMS. Actividad física. [Internet]. [Citado abril de 2018]. Disponible en: http://www.who.int/dietphysicalactivity/pa/es/

30. OMS. Inactividad física: un problema de salud pública mundial. [Internet]. [Citado abril de 2018]. Disponible en: http://www. who.int/dietphysicalactivity/factsheet_inactivity/es/

31. OMS. Recomendaciones mundiales sobre actividad física para la salud. 2010. [Internet]. [Citado abril de 2018]. Disponible en: http://whqlibdoc.who.int/publications/2010/9789243599977_spa.pdf

32. Espinoza L, Rodríguez F, Gálvez J, MacMillan N. Hábitos de alimentación y actividad física en estudiantes universitarios. Rev Chil Nutr. 2011;38(4):458-65.

33. Ratner R, Hernández J, Martel J, Atalah E. Calidad de la alimentación y estado nutricional en estudiantes universitarios de 11 regiones de Chile. Rev méd Chile. 2012;140(12):1571-9.

34. OMS. Fomento del consumo mundial de frutas y verduras. [Internet]. [Citado abril de 2018]. Disponible en: http://www.who. int/dietphysicalactivity/fruit/es/ 